\title{
THEORETICAL PRINCIPLES, PRESENT STATUS \\ AND PROSPECTS FOR DEVELOPMENT OF MATERIAL SEPARATION IN MAGNETIC FLUIDS
}

\author{
V.N. GUBAREVICH AND S.V. VIDSOTA \\ The State Design Institute Gipromaschugleobogaschenie, \\ 348000 Lugansk, Ukraine
}

(Received May 1, 1993, final revision January 27, 1994)

\begin{abstract}
This paper reviews theoretical principles of material separation in magnetic fluids according to their density. The main formulae and expressions are summarised and possible prospects for development of this relatively young branch of separation are indicated. Specifications are given for separators of this type, as designed over the last several years by the Lugansk Institute "Gipromashugleobogaschchenie". Practical experience obtained in the Institute is outlined.
\end{abstract}

\section{INTRODUCTION}

The idea of separation of materials according to their specific gravity became practicable at the end of the sixties when large quantities of relatively inexpensive and physically stable ferromagnetic fluids were synthesized. Previously, the technique was associated mainly with the so-called magnetohydrostatic separation; the method of separation of particles according to their density in water solutions of various paramagnetic salts [1-3].

In spite of numerous theoretical studies and laboratory developments this method was, however, far from practical application as a result of weak magnetic properties of paramagnetic solutions. Although the possibility of such a 
beneficiation was, in principle, well proven, this technique was too far from achieving necessary effective densities in the working volume of separation devices. Moreover, dimensions of working gaps between the poles of such separators were limited as a result of the need to maintain high intensity magnetic field in the separation volume (up to $20 \mathrm{kOe}$ ). Capacity of such devices was thus very small (up to 50 grams per hour).

Breakthrough was achieved when synthetic strongly magnetic fluids, the so-called ferrofluids, were prepared. Having high saturation magnetization (up to 50 Gauss), at relatively low viscosity (up to $5 \times 10^{-2}$ poise) they represented ideal medium for separation of materials according to their density in sufficiently broad limits (up to $\left.21 \mathrm{~g} / \mathrm{cm}^{3}\right)$.

Consequently, following numerous technical applications, studies of ferrohydrostatic separation began to develop rather rapidly as a particular method of beneficiation of raw materials in ferromagnetic fluids. The additional magnetobuoyant force which can be considered as effective density of medium was identified in a classical paper by Neuringer and Rosenzweig [4].

Rosenzweig further developed this field and contributed significantly to this branch of science - ferrohydrodynamics [5]. In 1969 he patented a method of material separation using ferromagnetic medium marking the beginning of numerous similar patents in the USA, Japan, Israel, France and USSR [6-9]. It should be noted that despite considerable progress for the last 25 years ferrohydrostatic separation has not emerged yet from its experimentally rudimentary state.

Numerous organisations dealing with this problem introduced prototypes of ferrohydrostatic separators (FHS) [10,11]; they were not, however, in a position to apply them on a wider production scale. On the other hand, Soviet researchers applied various prototypes on industrial scale. For instance, the Lugansk Institute "Gipromashugleobogashchenie" (Ukraine) has been, for the last 15 years, designing ferrohydrostatic apparatuses for coal preparation, the final concentration of diamonds and gold-bearing ores, as well as for separation of non-ferrous and semiprecious metals from scrap. 
In what follows, general theoretical review of experience accumulated during that period will be given and specifications of commercially available separators will be summarised.

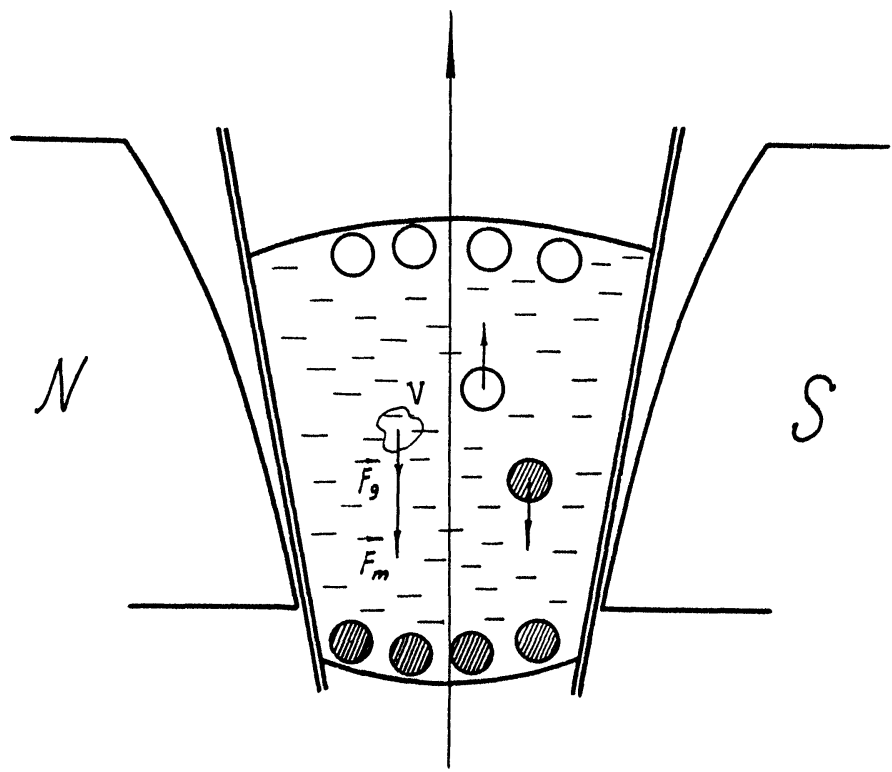

Fig. 1 Schematic diagram of ferrohydrostatic separation

General diagram of the process of ferrohydrostatic separation according to specific density is shown in Figure 1. The additional force $\mathbf{F}_{\mathrm{m}}$, equal to $\mathbf{M ~ V ~} \partial \mathrm{H} / \partial \mathbf{z}$ (where $\mathrm{M}$ is the specific magnetisation of medium) acts on a given volume $V$ of magnetic fluid in non-homogeneous magnetic field. It is assumed that $V$ is sufficiently small so that the variation of the gradient within the volume can be neglected. Thus, the total force acting on a given volume is equal to:

$$
\mathbf{F}=\mathbf{F}_{\mathrm{g}}+\mathbf{F}_{\mathrm{m}}=\mathrm{mg}+\mathrm{M} \frac{\partial \mathrm{H}}{\partial \mathrm{z}} \mathrm{V} \frac{\mathbf{g}}{\mathrm{g}}
$$

By expressing the mass $m$ as a function of density of medium $\rho_{0}$, eq. (1) can be rewritten as:

$$
\mathbf{F}=\left(\rho_{0}+\mathbf{M} \frac{\partial \mathrm{H}}{\partial \mathbf{z}} \mathbf{g}^{-1}\right) \mathbf{g} \mathbf{V}
$$


It can be seen that the expression in parenthesis can be viewed as an effective density of medium:

$$
\rho=\rho_{0}+\mathrm{M} \frac{\partial \mathrm{H}}{\partial \mathrm{z}} \mathrm{g}^{-1}
$$

Equation (3) is the main relationship on which the theory of ferrohydrostatic separation is based.

\section{CALCULATION OF THE WORKING VOLUME OF THE SEPARATOR}

The working volume of a ferrohydrostatic separator is the volume where separation of particles takes place. It is clear that the most important technological characteristics of such an apparatus depend on geometrical shape of the working zone and the design of a ferrohydrostatic equipment must, therefore, be based on the calculation of the working zone. In principle, it is a simple task and can be reduced to finding distribution of the magnetic field in the inter-pole gaps of different shapes.

A large variety of methods and techniques of calculation of the magnetic field distribution can be found in relevant literature. Unfortunately, many authors use complicated mathematical calculations although these are not always needed. Any real magnetic field $\mathbf{H}$ must satisfy Maxwell's equations:

$$
\operatorname{div} \mathbf{H}=0 \quad \text { and } \quad \operatorname{curl} \mathbf{H}=\mathbf{0}
$$

Physical conditions in the inter-pole gap concern static magnetic field in which the electric current and charges are absent. The second equation in (4) is mathematically equivalent to the requirement that vector $\mathbf{H}$ could be expressed as a gradient of a scalar function $U=U(\boldsymbol{r})$, namely

$$
\mathbf{H}=-\operatorname{grad} \mathrm{U}
$$

System of equations (4) can thus be replaced by a single Laplace equation:

$$
\Delta \mathrm{U}=0
$$


The function $U=U(x, y)$ (in practice we are always concerned with two-dimensional problems) which represents the solution of eq. (6) gives a grid of mutually orthogonal curves one of which forms a set of force lines and the other a set of equipotential lines of a given magnetic field. So it appears that if surfaces of magnetic poles coincide with surface of equipotentials then the magnetic field (5) will be created in the inter-pole gap.

We can thus see that the objective of all mathematically rigorous calculations is to find such solutions of eq. (6) equipotential surfaces of which would be acceptable as boundary conditions. Solution of the Laplace equation is a rather complicated task. The general solution has been developed only recently and several classes of special analytical functions have been introduced. Consequently, all our "calculations" are essentially nothing else than a selection of existing solutions for a particular kind of boundary conditions. As a rule, the problem always concerns the shape of the inter-pole gap.

If equipotential curves fit the surface of magnetic poles sufficiently well it is possible to say that we succeeded in "calculating" the magnetic field. This is possible, however, in a limited number of special cases only. Moreover, one still has to guess whether the given inter-pole gap corresponds to a certain harmonic function satisfying the Laplace equation. This can be easily done, for instance, for wedge-shaped inter-pole gap (Fig. 2a). It can be seen that, in polar coordinates, the lines forming the surface of the poles are given by a function $\varphi=$ const. which satisfies equation (6).
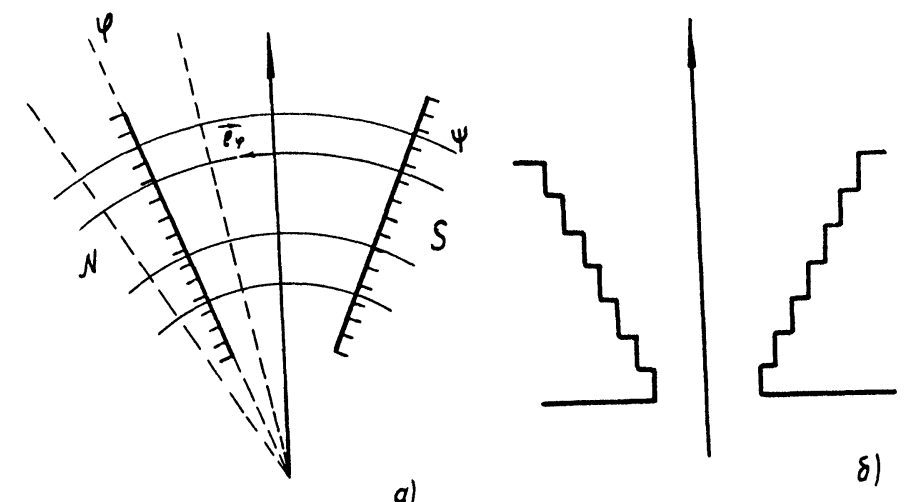

Fig. 2 A wedge-shaped interpolar gap as used to calculate magnetic field distribution in the working space. 
Assuming that $V=A \varphi$, where $A$ is a constant, we find $\mathbf{H}=-\frac{\mathbf{A}}{\mathbf{r}} \mathbf{e}_{\varphi}$ which represents the solution of our problem. In most cases, however, it is impossible to solve the problem for a gap shown in Fig. $2 \mathrm{~b}$ and numerical methods must be used. Their popularity can be explained by principal possibility of solving the Laplace equation with boundary conditions of any predetermined form, even in those cases where analytical solution is impossible.

Nevertheless, it cannot be claimed that numerical methods fully exclude analytical methods. Although numerical methods have a number of advantages their main drawback is that apart from being cumbersome they do not allow a general analysis to be carried out which is often necessary in applied engineering and technical calculations. Moreover, we assume that the very logic of solving the problem requires primarily the knowledge of the form of the magnetic and of its gradient, with subsequent calculation of the shape of the inter-pole gap which generates the given form of the field.

We feel, therefore, that it is advisable to concentrate on analytical methods. From a wide array of magnetic potentials $U$ it is possible to single out the one that is most suitable for the solution of the problem, for instance:

$$
\mathrm{U}(\mathrm{x}, \mathrm{y})=\mathrm{C} \mathrm{e}^{\alpha \mathrm{y}} \sin \alpha \mathrm{x}
$$

It can be easily found that function (7) satisfies eq.(6) and is therefore a harmonic function. The pole curve is in this case given by:

$$
\mathrm{e}^{\alpha \mathrm{y}} \sin \alpha \mathrm{x}=\text { const }
$$

With the ordinate axis directed vertically down, as is shown in Fig. 3 the magnetic field in the inter-pole gap can be easily calculate using eq. (5):

$$
\mathbf{H}=-\alpha \mathrm{Ce}^{\alpha \mathrm{y}}(\mathrm{i} \cos \alpha \mathrm{x}+\mathrm{jsin} \alpha \mathrm{x})
$$

By defining the value of the magnetic field at the origin as $H_{0}$ we can find the value of constant $C$. Thus, the magnetic field strength on the symmetry axis of the inter-pole gap, as defined by eq. (8) is given by: 


$$
\mathbf{H}=\mathrm{H}_{0} \mathrm{e}^{\alpha \mathbf{y}} \mathbf{i}
$$

while the module of the gradient is equal to:

$$
\frac{\partial \mathrm{H}}{\partial \mathrm{y}}=\alpha \mathrm{H}_{0} \mathrm{e}^{\alpha \mathrm{y}}
$$

Equations (10) and (10') contain a parameter $\alpha$ on which the value of the effective density of the FHS separator mainly depends. Indeed, substituting eq. (10') into eq. (3) we obtain:

$$
\rho=\rho_{0}+\frac{\mathrm{M} \alpha \mathrm{H}_{0} \mathrm{e}^{\alpha \mathrm{y}}}{\mathrm{g}}
$$

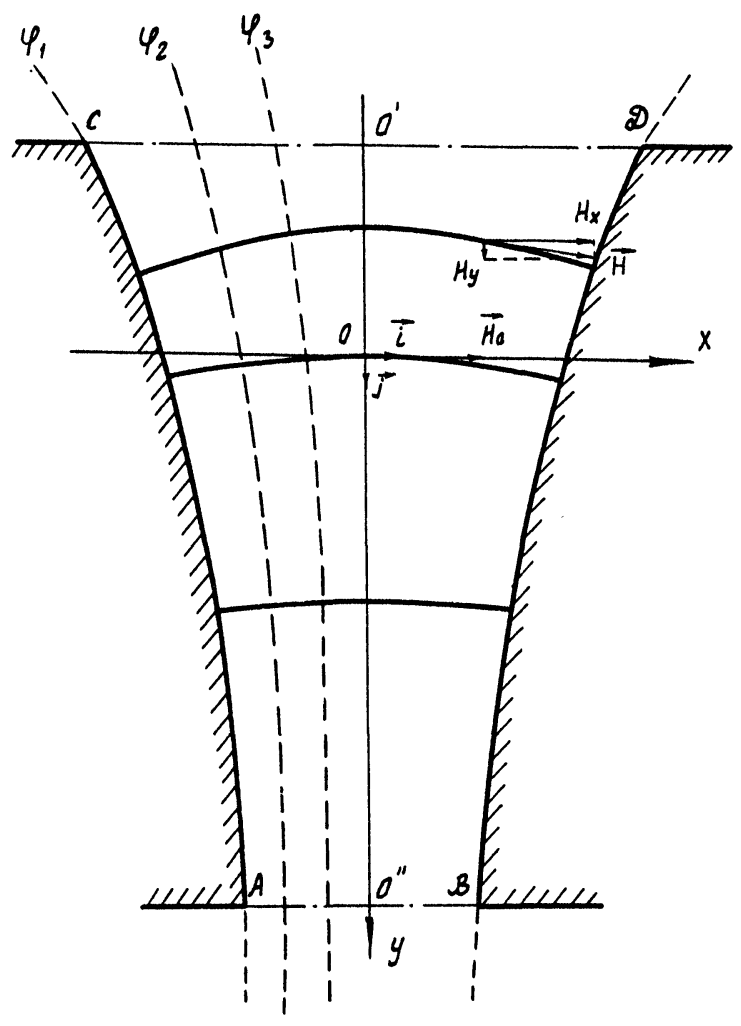

Fig. 3 An interpolar gap of logarithmic shape as used to calculate magnetic field distribution in the working space. 
By increasing $\alpha$ and simultaneously varying the shape of the pole gap according to eq. (8) we can expect to obtain any required value of the effective density. As a result of the presence of the exponential factor $e^{\alpha y}$ in eq. (11) a large nonuniformity of density over the height of the gap appears. Thus, in practice, the value of $\alpha$ should not be too large and it usually does not exceed $0.1 \mathrm{~cm}^{-1}$.

For a sufficiently small $\alpha$ the exponent in eq. (11) can be expanded into series and as an approximation, only the first two terms can be retained. Under these conditions the expression (11) can be rewritten:

$$
\rho=\rho_{0}+\frac{\mathrm{M} \alpha \mathrm{H}_{0}}{\mathrm{~g}}+\frac{\mathrm{M} \alpha^{2} \mathrm{H}_{0}}{\mathrm{~g}} \mathrm{y}
$$

It is now obvious that the analytical condition of nonuniformity of the effective density in the working zone of the inter-pole gap is represented by the last term in eq.(12). If $h$ is a typical height of the working zone then the sharper the inequality (13) is, the larger is the nonuniformity of the effective density:

$$
\frac{\mathrm{M} \alpha^{2} \mathrm{H}_{0}}{\mathrm{~g}} \mathrm{~h} \ll 1
$$

For a typical case of $M \approx 20$ Gauss, $H_{0} \approx 5 \mathrm{kOe}$ and $h=5 \mathrm{~cm}$ we obtain, from eq. (13):

$$
\alpha \ll\left[\frac{\mathrm{y}}{\mathrm{MH}_{0} \mathrm{~h}}\right]^{\frac{1}{2}} \approx 0.04 \mathrm{~cm}^{-1}
$$

For such a typical value of $\alpha \approx 10^{-2} \mathrm{~cm}^{-1}$ the effective density $\rho \approx 2$ to $2.5 \mathrm{~g} / \mathrm{cm}^{3}$ will be created in the working zone. It can be increased either by increasing $\alpha$ (which would result in deterioration of technological parameters of separation) or by increasing $\mathrm{H}_{0}$ which is, of course, undesirable in view of increasing technical difficulties.

Finally, in each case we have to choose between quantity and quality: either we strive to increase a high density and sacrifice selectivity of the FHS separator or we can obtain highly selective separation at the cost of relatively low effective density in the working zone. In principle it is probably impossible to design an apparatus operating efficiently in the entire range of variation of $\rho$. 
The method of calculation of the inter-pole gap proposed here is certainly not the only one but it has been found to be optimum when applied to a wide range problems in ferrohydrostatic separation. Whether to use it or to replace it by any other similar approach depends exclusively on characteristic features of a given separation problem. The final approach taken will be strongly affected by intuition of a designer.

\section{MAGNETIC FLUIDS AS A SEPARATION MEDIUM}

From the point of view of ferrohydrostatic separation, the stability, the magnetisation and the viscosity in external magnetic field are the most significant physical properties of magnetic fluids.

The stability is associated with experimentally observed ability of magnetic fluids to undergo various structural conversions and phase transformations, as a function of time, both under the exposure to a magnetic field and in its absence. There is no complete universally recognised theory of magnetic fluids that would reliably describe phenomena usually observed in these fluids. Instead, experimental data have been systematically accumulated and theoretical models, frequently contradicting each other have been developed. These models usually describe various properties of magnetic fluids without treating their common structure. It is, however, clear that physical properties of ferrofluids depend considerably on the techniques of their preparation.

It is accepted that it is, in principle, possible to produce stable, non-stratified and resistant to phase changes magnetic fluids in large volumes. These magnetic fluids are suitable for a long-term operation in FHS separators. Practicalities of their production are beyond the scope of this paper.

We shall assume that such fluids are available and that they meet the requirement of long-term stability. The other important property of magnetic fluids is their ability to be magnetised in external magnetic field. It was particularly this property that determined a rapid growth of numerous technical applications of these fluids in the last twenty years. 
It can be seen from eq.(3) that the effective density $\rho$ is directly proportional to magnetisation $M$ and therefore, the higher the magnetisation, the higher effective density can be obtained. In general, in the first approximation, the magnetisation is given by the Langevin function

$$
\mathbf{M}=\mathbf{M}_{\mathbf{s}}\left(\operatorname{coth} \mathbf{x}-\frac{1}{\mathrm{x}}\right)
$$

where $\mathrm{x}=\frac{<\mu>\mathrm{H}}{\mathrm{k} \mathrm{T}},<\mu>$ is the mean magnetic moment of particles of disperse phase, $M_{\mathrm{s}}=\mathrm{n}\langle\mu\rangle$ is the saturation magnetisation and $n$ is the volumetric concentration of particles. The saturation of the above mentioned function takes place at relatively weak magnetic fields (up to 1 to $2 \mathrm{kOe}$ ). The saturation is enhanced by the presence of interpole interactions in the disperse phase of the ferrofluid and statistical scatter of the value of $\mu$. Equation (14) thus gives a conservative value of $M=M(H)$.

As a result of the fact that typical values of the magnetic field strength in the working zone of a separator are usually much higher than 1 or $2 \mathrm{kOe}$, the function (14) can be simplified in the following way:

$$
M=M_{s}\left(1-\frac{1}{x}\right)
$$

which is valid for $x \gg 1$. In practice, in all preliminary calculations, this function can be replaced by $M_{\mathrm{s}}$. Validity of such a simplification depends on the type of the problem being solved. If a calculation of desired effective density in the working zone of an apparatus to be designed is to be performed, then the replacement of eqs. (14) and (14') by the value of $M_{\mathbf{s}}$ is fully justified.

If, however, the bottom boundary $S$ is to be estimated, particularly in the case of a suspension layer of ferromagnetic fluid in the inter-pole gap, then the knowledge of of the dependence of $M$ on $H$ is required. There is a variety of information in the literature on the saturation magnetisation of ferrofluids and Figure 4 shows the dependence of the saturation magnetisation on the density of the ferromagnetic fluid, based on data obtained from various sources $[12,13]$. These values are perfectly suitable for preliminary calculations and can be used in relations (14) and $\left(14^{\prime}\right)$. 


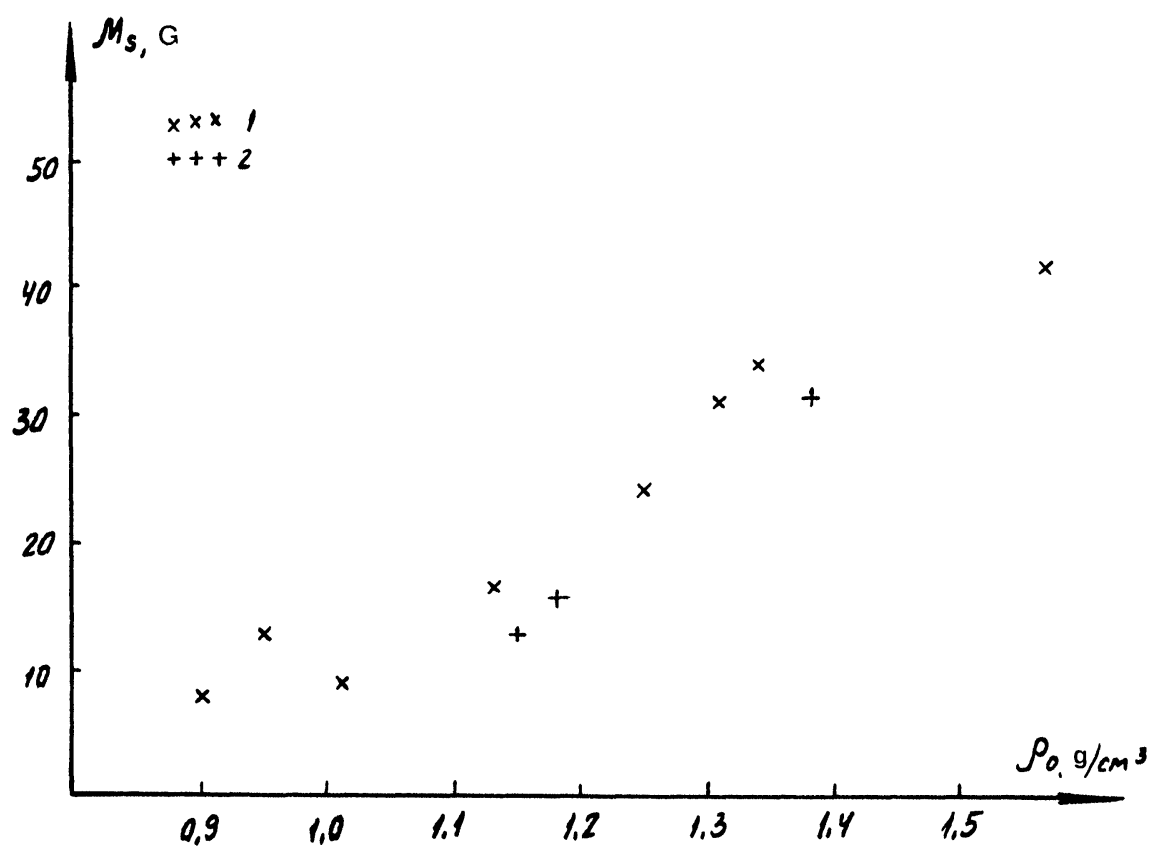

Fig. 4 Dependence of saturation magnetisation of a ferrofluid on physical density: 1-kerosene-based ferrofluid, 2-water-based ferrofluid

Another important property of magnetic fluids is the dependence of their viscosity on external magnetic field. In the absence of the external magnetic field the ferrofluid represents a concentrated suspension the viscosity of which can be described by the Wand relation:

$$
\eta=\eta_{0} \exp \frac{2.5 \mathrm{n}+2.7 \mathrm{n}^{2}}{1-0.609 \mathrm{n}}
$$

where $\eta$ and $\eta_{\mathrm{o}}$ are the dynamic viscosities of the colloid and fluid phases, respectively, and $n$ is the volumetric concentration of the solid phase.

When the ferrofluid is exposed to magnetic field, the elementary dipoles rotate and capture particles of the disperse phase and the effective viscosity increases. It is 
assumed that the rotating (effective) viscosity of the magnetic fluid $\eta_{\mathbf{H}}$ is proportional to its viscosity $\eta$ in the absence of the field. For the mean concentration of ferromagnetic fluid (with $n \approx 0.03$ to 0.2 ), the limiting viscosity in the magnetic field can be estimated from the relation:

$$
\eta_{\mathbf{H}}=\eta\left(1+\frac{2}{3} \mathrm{n}^{\prime}\right)
$$

where $n^{\prime}$ is the hydrodynamic concentration. Hydrodynamic concentration is related to the volume occupied by solid particles in a ferromagnetic fluid, taking into account the particles are covered by a coat of molecules adsorbed onto their surface and having an appearance of a liquid. Thus:

$$
\mathrm{n}^{\prime}=\frac{\mathrm{V}^{\prime}+\mathrm{V}^{\prime \prime}}{\mathrm{V}} \text { and } \mathrm{n}=\frac{\mathrm{V}^{\prime}}{\mathrm{V}}
$$

where $V$ is the total volume of the liquid, $V^{\prime}$ is the volume of solid phase and $V^{\prime \prime}$ is the volume of the adsorption layer that covers the surface of the particles.

Figure 5 shows experimental data of the dependence of viscosity of kerosene-based ferrocolloids with magnetite disperse phase on density of the magnetic fluid $\rho$.

Measurements indicate that the effective viscosity of ferrofluids does not practically increase with increasing magnetic field for magnetic field higher than $4 \mathrm{kOe}$. Typically, the magnetic field strength in the working zone of FHS separators is never lower than this value. We can thus consider the viscosity of magnetic fluids in the entire volume of the inter-pole space to be constant.

It should be noted that production of large volumes of ferrofluids in industrial conditions is accompanied, as a rule, by considerable variations in their physical properties, including viscosity. It is caused by the presence of high concentrations of large particles and of aggregates of the disperse phase. Removal of such anomaly large particles, e.g. by mechanical centrifuging, changes slightly the density and the magnetisation of the ferrofluid, but sharply improves its viscosity properties. The dependence of $\eta$ on prehistory of the sample and on the duration of its storage disappears. 


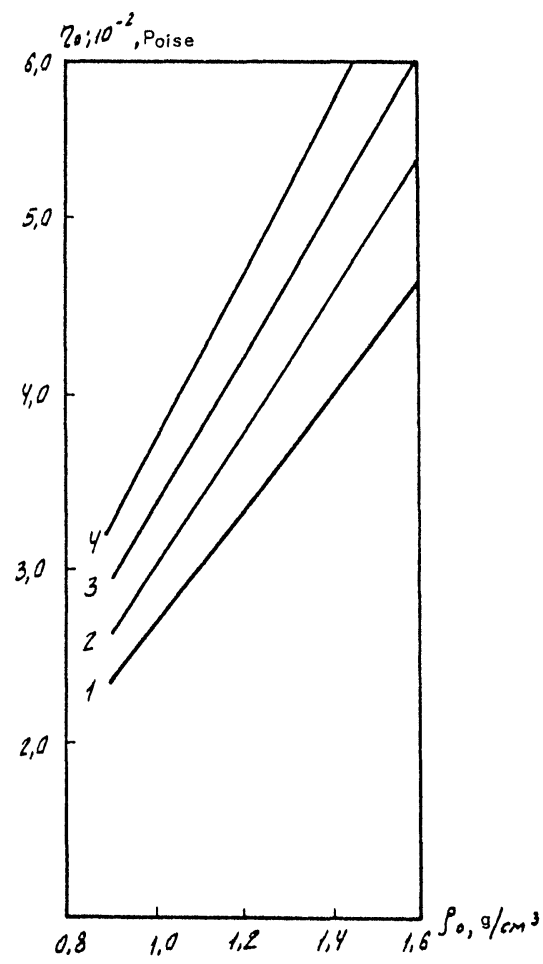

Fig. 5 Dependence of viscosity of magnetic fluid on its density, for kerosene viscosity $1.75(1), 2.0(2), 2.25(3)$ and $2.5(4)$

\section{HYDROSTATICS OF A MAGNETIC FLUID IN THE SEPARATOR}

The hydrostatics of the magnetic fluid in the inter-pole gap of a ferrohydrostatic separator is of interest because the separation of a raw material in a suspended layer of the ferromagnetic fluid is technically the most comfortable method of such a separation. Diagram of approximate location of the fluid in the gap is shown in Fig. 6a. After entering the zone of separation, heavy fraction of a material to be separated settles through the fluid layer and after leaving the fluid it can be easily removed from the separator. If a container with solid bottom (Fig. 6b) is used it would be necessary to apply mechanical vibrations or to provide special discharge device to remove the material from the system. It should be noted, however, that with decreasing size of raw material the value of the viscosity forces 
increases considerably and the vibration discharge for very small particle sizes is not possible.

Moreover, the presence of mechanical vibrations in the separation medium will negatively affect technological parameters of separation. Basic drawback of the above described method of retention of the magnetic fluid in the working zone of the separator is considerable sagging of the fluid layer. Under such circumstances a considerable portion of the fluid (sometimes up to one half of the total volume) can be found outside the working zone. The following theoretical analysis was developed in order to investigate conditions of retention of the magnetic fluid in the inter-pole gap of the ferrohydrostatic separator.
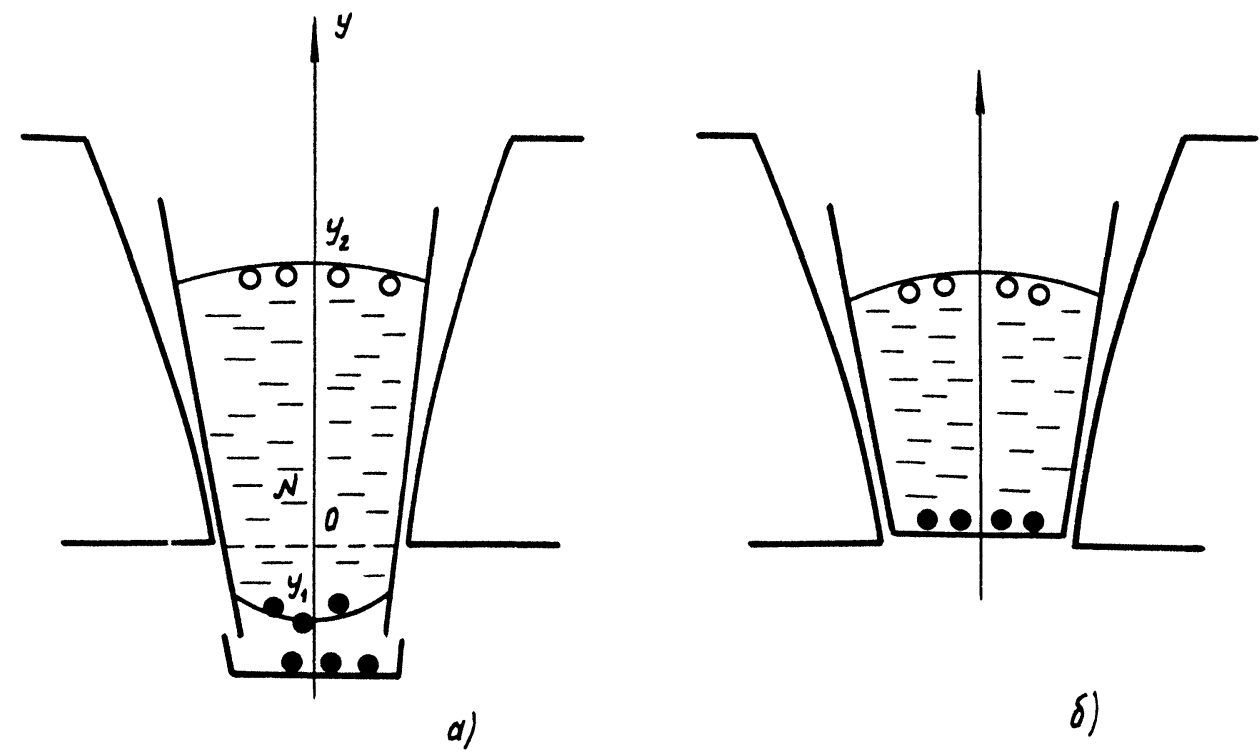

Fig. 6 Position of a magnetic fluid in the inter-pole gap of a ferrohydrostatic separator. a/ a suspended layer, b/ in a tray with solid bottom

Let us consider a one-dimensional situation assuming that the curvature of magnetic force lines can be neglected. In other words, instead of the entire layer of the ferromagnetic fluid we shall consider only a column of the liquid along the ordinate. It is further assumed that the transverse change of the magnetic field 
(and of its gradient) along its cross-section is sufficiently small. We have thus simplified our problem without a major detriment to the final results.

The hydrostatic equation can be written, for our case, as follows:

$$
\operatorname{grad} p=\mathbf{f}_{\mathrm{g}}+\mathbf{f}_{\mathrm{m}}
$$

where $f_{g}=\rho_{0} g$ is the specific weight, $f_{m}=M \frac{\partial \mathbf{H}}{\partial y}$ is the specific magnetic force and $p$ is the pressure of the ferrofluid. Integrating eq. (17) along OY from $\mathrm{y}_{1}$ to $\mathrm{y}_{2}$ we obtain:

$$
p\left(y_{2}\right)-p\left(y_{1}\right)=-\rho_{0} g \ell+\int_{H\left(y_{1}\right)}^{H} M d H
$$

where $\ell=\mathrm{y}_{2}-\mathrm{y}_{1}$ is the height of the column (or the thickness of the layer) of the ferromagnetic fluid in the gap. Taking into account that the pressures from both sides are equal, the left-hand side of eq. (18) is equal to zero, so that

$$
-\rho_{0} \mathrm{y} \ell+\int_{\mathrm{H}\left(\mathrm{y}_{1}\right)}^{\mathrm{H}\left(\mathrm{y}_{2}\right)} \mathrm{M} \mathrm{dH}=0
$$

Eq. (19) can be solved if the distribution function $H=H(y)$ is known. The problem, however, is that the suspended layer of ferrofluid is placed in the zone of end effects so that analytical determination of the required function is not possible. We have thus developed an empirical graphical technique of solving eq.(19) based on measurements of the magnetic field strength along the axis of symmetry of the inter-pole gap.

Fig. 7 depicts a typical graph of function $H=H(y)$. The selection of the zero point on the ordinate is of no importance because in what follows we shall be dealing only with differences in coordinates of the upper and lower ends of the ferrofluid column. 
In order to demonstrate the technique let us place the zero point at the lower edge of the pole pieces. Magnetic field in the inter-pole space and in its vicinity is usually sufficiently large so that we can neglect the dependence of $M$ on $H$, so that $M=M_{\mathrm{s}}$ in the first approximation. If necessary, however, the calculation can be made more precise replacing $M_{\mathbf{S}}$ by the mean value of magnetisation at $H \approx H_{1}-H_{2}$.

Eq. (19) can be easily rewritten as:

$$
\frac{\Delta \mathrm{H}}{\ell}=\frac{\rho_{0} \mathrm{~g}}{\mathrm{M}}
$$

where $\Delta H=H\left(y_{2}\right)-H\left(y_{1}\right)$.

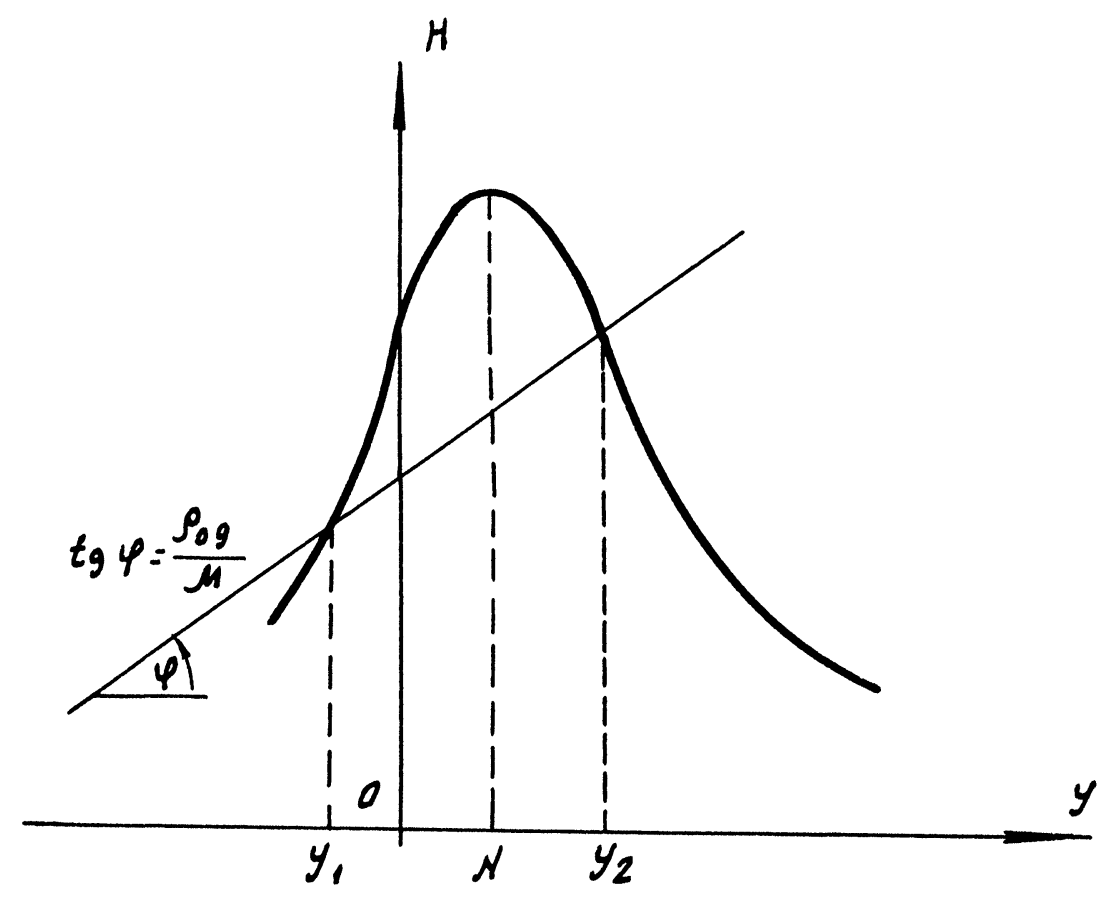

Fig. 7 Calculation of the hydrostatic equilibrium layer of a magnetic fluid 
According to eq. (20) abscissae of the cross points of the straight line $\mathrm{H}=\frac{\rho_{0} \mathrm{~g}}{\mathrm{M}} \mathrm{y}$ with curve $H=H(y)$ will give us the coordinates $y_{1}$ and $y_{2}$ of the corresponding ends of the column of the ferromagnetic fluid in the gap. It is clear that by a parallel transposition of this straight line it is possible to find a position of the sagging of the layer of the ferrofluid of a given thickness.

The proposed method of calculation is, however, suitable only for experimentally determined shapes of the inter-pole gaps. It is frequently quite sufficient since a small change in the magnetic pole gap does not affect substantially the dependence of the magnetic field strength on coordinates and it is thus possible to perform the calculation based on analogy when, in the first approximation, the known curves are used.

The investigation shows that the use of a suspended layer is particularly effective in gaps with a small gradient (i.e. for sufficiently small $\rho$ ), otherwise the squeezing out of ferrofluid becomes so large that in practice the working zone cannot be filled up. But even in favourable cases when the gradient is small, the sagging of the column of the fluid is, as a rule, still too large.

We proposed, as one of the possible methods to solve this problem, a technique of capture of the magnetic fluid in the gap of a FHS separator using the so-called "liquid bottom". Principle of this technique is based on adding a certain quantity of a strongly magnetic fluid based on a different liquid into the original ferrofluid. For instance, a ferrofluid based on kerosene was added into a water-based magnetic fluid.

When the working zone of a separator is filled with a such two-component mixture the strongly magnetic fluid will form a support (liquid bottom) on which the ferrofluid with required physical properties will be placed. Strong magnetisation of the support can hold a sufficiently high column of the working ferromagnetic fluid which thus does not slump too low. Free discharge of the heavy fraction of a separated raw material through the supporting layer is thus ensured, as a result of the fact that it can be positioned in the zone with reversed gradient (in Fig. 7 this area is to the left from point $N$ ). 
The above proposed method of calculation can also be extended to two-component ferrofluid systems; knowing magnetizations $M_{1}$ and $M_{2}$ of both components of the mixture, as well as the ratio of their volumes it is possible, in principle, to determine a position of the hydrostatic equalisation of such a binary column of ferromagnetic fluids in the inter-pole zone. And, at the same time, it is easy to calculate the quantity and the magnetisation of the supporting fluid required to capture a given layer of the working ferrofluid in a given area of the gap.

Preliminary evaluation shows that the sagging can be, in this case, reduced by a factor of at least 2 to 2.5. We are not going to present the relevant calculations since the idea is evident and quantitative calculations can be performed only using specific parameters of the magnetic system of a separator. We shall note only that such calculations are necessary when designing the so-called FHS regenerators, that is devices used to recover magnetic fluids from their mixtures with water. This necessity is associated with the problem of regeneration of the fluid which is carried out of the separation zone together with the separated material.

When a ferrofluid passes through the working zone it should be washed on special screens with a water solution of surface active substances. A fluid thus obtained is fed into a ferrohydrostatic regenerator where the capture of a magnetic component takes place. Investigations show that the recovery of a magnetic fluid in such regenerators exceeds $95 \%$ of the ferromagnetic fluid entering the regenerator.

\section{PROSPECTS OF FERROHYDROSTATIC SEPARATION}

The possibilities of ferrohydrostatic separation as a special branch of material treatment depend on how soon its specific features will be developed. Necessity of using large quantities of magnetic fluids as well as technological and ecological difficulties associated with these fluids will probably not allow this technique to be applied as widely as, for instance, flotation.

Generally speaking, it is, however, not necessary. The main area of application of ferrohydrostatic separation is the processing of municipal and industrial wastes. For instance, France produces 568 million tonnes of waste annually: 18 million 
tonnes of municipal waste, 150 million tonnes of industrial waste and 400 million tonnes of agricultural waste. Secondary resources supply one third of requirements of raw materials in French industry.

It is hoped that a wide introduction of ferrohydrostatic separation will increase this share considerably. One possible area of application of ferrohydrostatic separation is, for example, separation of cables and of radioelectronic and motor industry scrap. Our studies show that the recovery of pure metal from copper-aluminium and lead-copper mixtures by ferrohydrostatic separation can be as high as $99 \%$ without a special financial outlay. Taking into account high prices of pure metals at the present world market the capital cost of ferrohydrostatic equipment can be recovered in less than a year keeping in mind that in car manufacturing areas considerable amount of automobile scrap is being accumulated which can be reprocessed only by ferrohydrostatic technique.

Particularly efficient application of FHS is the retreatment of gold-platinum and diamond concentrates. The operational experience at the Norilsk mining and metallurgical plant shows that one pass of a material containing precious metals through a ferrohydrostatic separator can increase the concentration of PGMs from 3 to $4 \%$ to 60 to $70 \%$. The mine tailings can thus be economically processed. Purely from technical point of view the recovery of these metals from ore by FHS is a viable process since the metals are rather heavy while the density of the feed material is usually lower (about $5 \mathrm{~g} / \mathrm{cm}^{3}$ ).

An equipment with low resolution but with high densities required for the recovery of small quantities of heavy fractions from large volumes of raw material can thus be designed. The high price of the final concentrates (particularly those containing precious metals) will result in repayment of the capital cost in a short time.

The recovery of diamonds by ferrohydrostatic processing can be effective using a high-resolution FHS separator which does not present a difficulty in view of relatively low density of diamonds.

Apart from obvious advantages of the ferrohydrostatic separation there are several drawbacks. The most important ones are the high cost of large volumes of high-quality magnetic fluid and environmental impact of using such large 
quantities. Although recent research and development of physics of magnetic fluids reduced the negative impact of this technique, numerous problems still remain.

Further research is stimulated by the fact that ferrofluids are finding new applications in various technical devices, e.g. ink for high-speed printers. It is, therefore, hoped that a major breakthrough will be achieved in a short time.

Experimental results of ferrohydrostatic separation of coal, ores and metals in magnetic fluids can be found in [13]. The specifications of some of the FHS separators designed and manufactured by the Lugansk Institute "Gipromashugleobogashchenie" are listed in Table I. and a photograph of the FHS-40 is given in Figure 8.

\section{NOMENCLATURE}

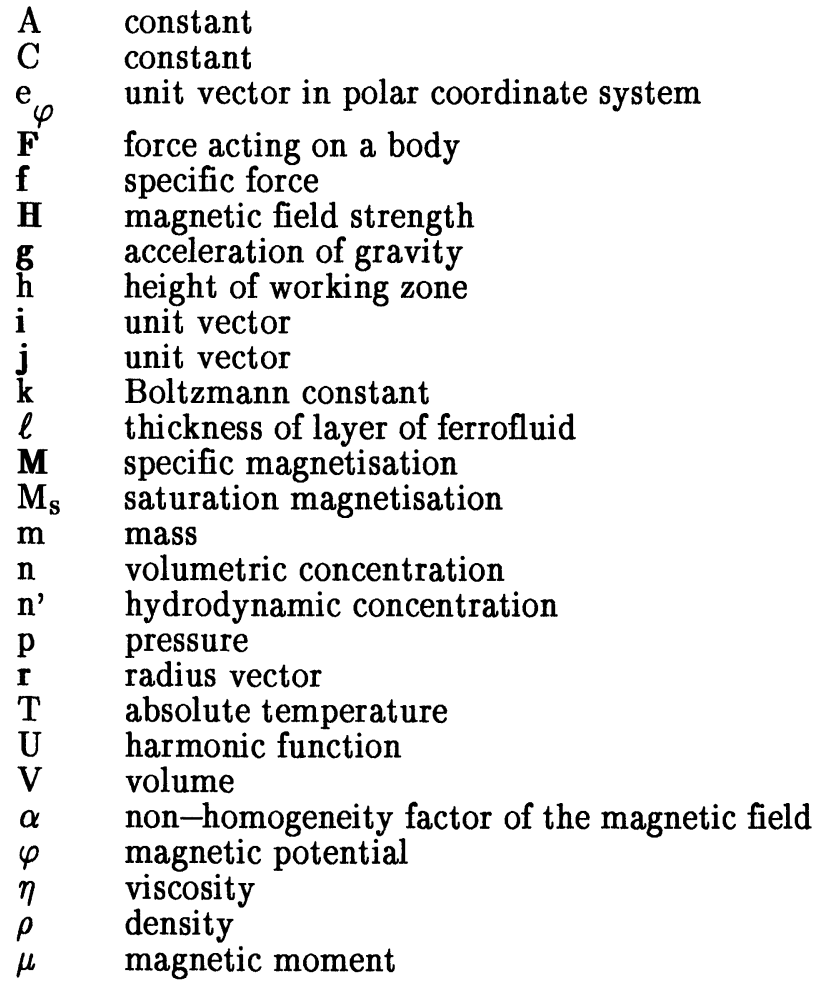




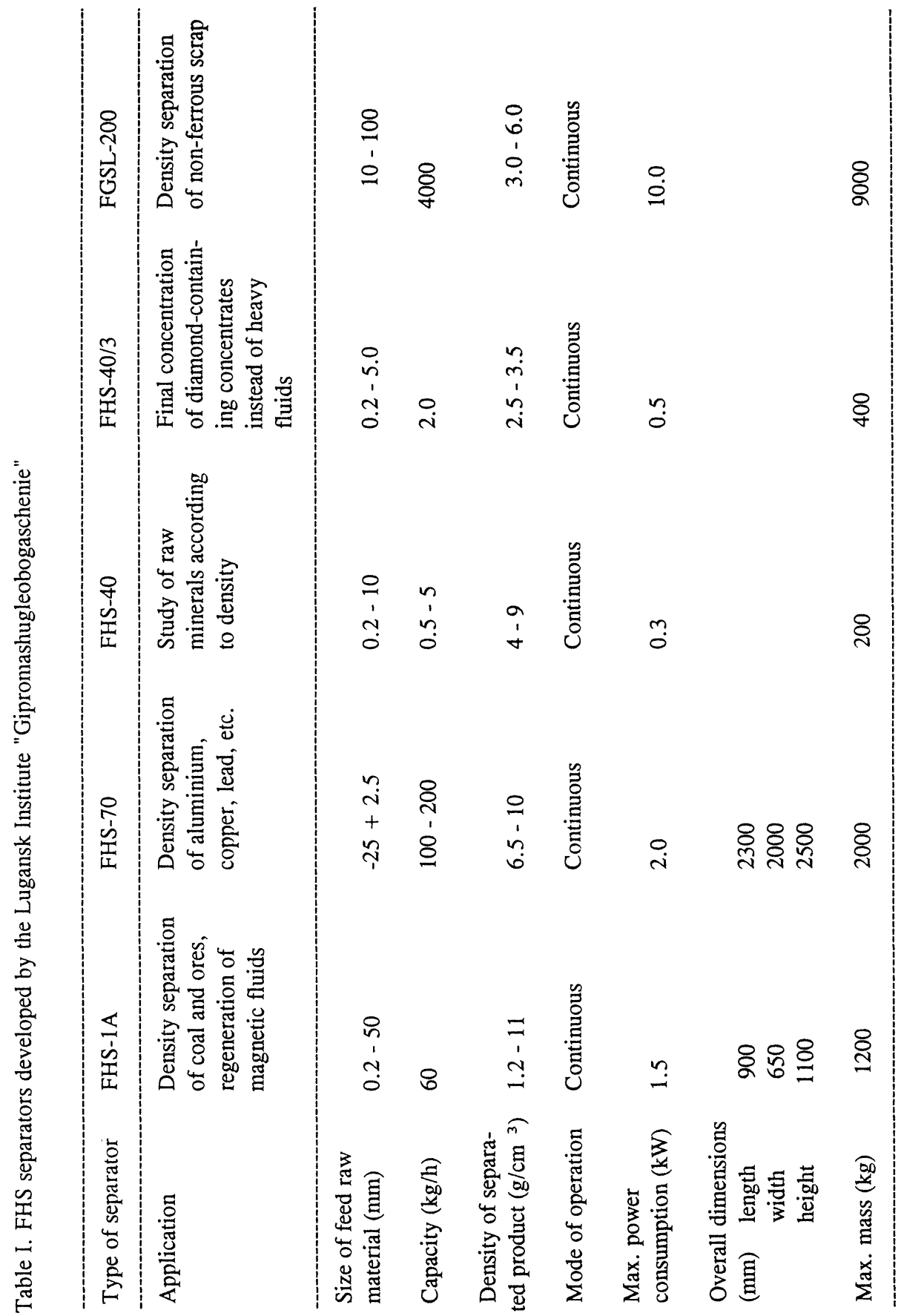




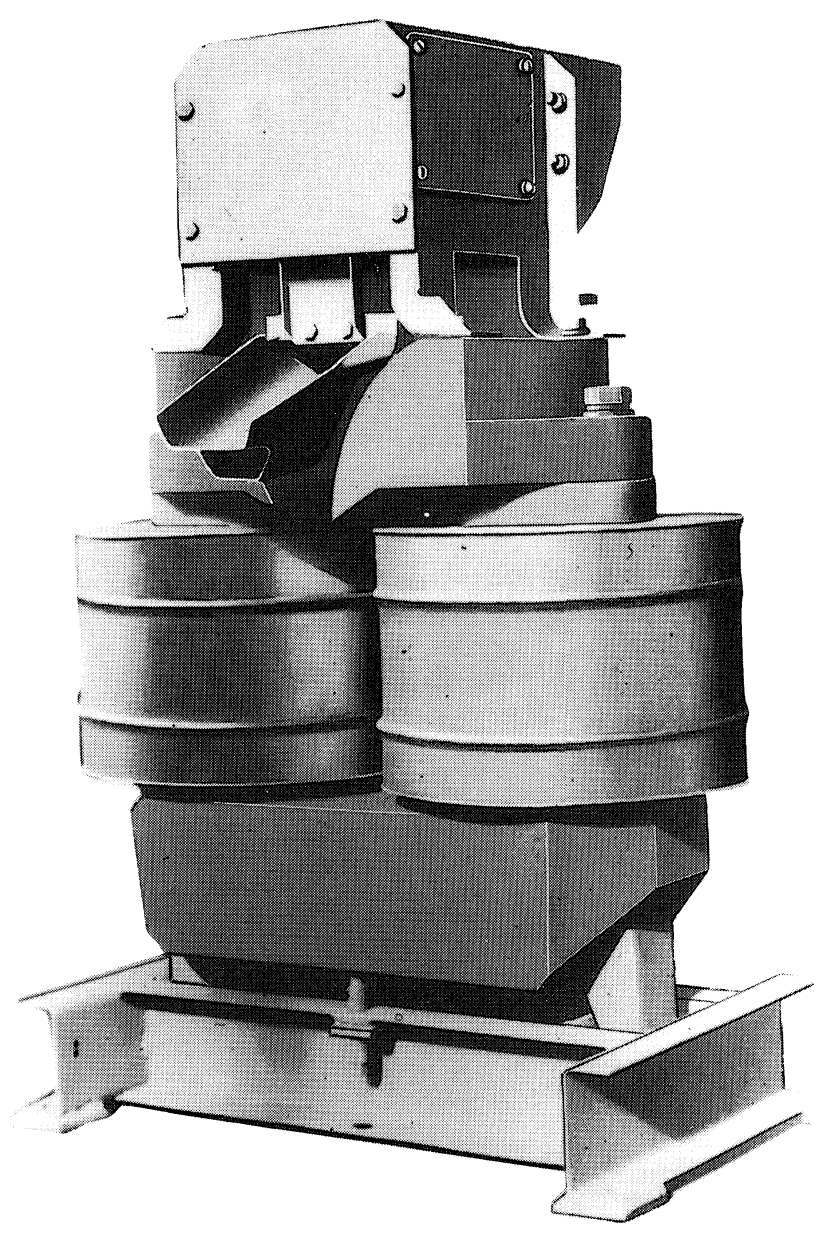

Fig. 8. Ferrohydrostatic separator FHS -40 


\section{REFERENCES}

[1] G.M. Bunin and U.C. Andres: Obogashchenie Rud no. \& (1966), 24 (in Russian)

[2] A.I. Berlinski et al.: In Laboratory and technological studies and methods of processing raw materials, Nedra Moscow (1975), p. 53

[3] M. Chiotti: Boll. Assoc. Miner. Subalpina 5 (1968), 700

[4] J.L. Neuringer and R.E. Rosenzweig: Phys. Fluids 7 (1964), 1927

[5] R.E. Rosenzweig: Ferrohydrodynamics, Cambridge University Press, Cambridge 1985

[6] Magnetogravimetric separator. US Patent 2265458 (1974)

[7] Apparatus for separation of non-magnetic materials. Japanese Patent No. 459441 (1977)

[8] Magnetic separator with hyperbola poles. French patent 2231431 (1974)

[9] Magnetohydrostatic separator. Author's Certificate (USSR) No. 671848 (1979)

[10] T. Fujita et al.: An improved sink-float resting apparatus for coal preparation using a water-based magnetic fluid, In: Proc. 11th Internat. Coal Prepar. Congress, Tokyo, Japan (1990)

[11] R.A. Khlebnikov: Elektron. Tech. 7(1970), 99 (in Russian)

[12] A.I. Berkovski et al.: Magnetic Fluids. Chimiya, Moscow 1989 (in Russian)

[13] V.N. Gubarevich: Separation of materials in magnetic fluids. Nedra, Moscow, 1987 (in Russian)

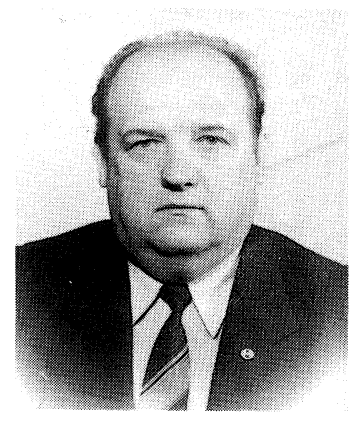

Vladimir Nikolaevich GUBAREVICH was born in 1939 and graduated from the Moscow Institute of Radiotelectronics and Mining Electromechanics. Dr. Gubarevich's main interest is the design of magnetic separators and devices for separation of non-magnetic metals and ores and concentration of diamond-bearing raw materials in magnetic fluids $\mathrm{He}$ is an author of 110 inventions and patents and of 45 papers. 




Sergei Viktorovich VIDSOTA was born in 1961 in Lugansk and graduated in theoretical physics from Donetsk State University $\mathrm{He}$ is presently involved in research into magnetic fluids and magnetic fields. He is an author of several papers.

Keywords: ferrofluid, magnetic separator, magnetisation, density 